INPLASY

PROTOCOL

To cite: Fu et al. Effectiveness and safety of acupuncture for rectal prolapse: a protocol of systematic review and metaanalysis. Inplasy protocol 2020100074. doi:

10.37766/inplasy2020.10.0074

Received: 20 October 2020

Published: 21 October 2020

Corresponding author:

Yong Tang

30534764@qq.com

Author Affiliation:

Jiangxi University of

Traditional Chinese Medicine

Support: NNSF(CN):81760787.

Review Stage at time of this submission: The review has not yet started.

Conflicts of interest:

The authors declare no conflicts of interest.

\section{Effectiveness and safety of acupuncture for rectal prolapse: a protocol of systematic review and meta-analysis}

Fu, W1; Tang, Y2; Yi, S³.

Review question / Objective: To evaluate the effect of acupuncture versus non-acupuncture treatment for rectal prolapse patients.

Condition being studied: Rectal prolapse is a kind of anorectal, rectal and partial sigmoid colon prolapse out of the anus disease, prone to children and the elderly, clinical incidence is not high, but because it is often accompanied by bleeding, edema, resulting in patients with fatigue, dizziness, and even incontinence, seriously affecting the quality of work and life.At present in view of the Rectal prolapse III degree and above, with the major surgery, and for Idegree, II degree mainly uses the conservative treatment in patients with Rectal prolapse. As western medicine has no specific treatment drugs, Treatment is symptomatic treatment, so the efficacy is poor, and the recurrence rate is high. Traditional Chinese medicine (TCM) has rich experience in treating Rectal prolapse, so many patients begin to seek TCM treatment. With the application of clinical practice, acupuncture, as a traditional green therapy, not only has remarkable curative effect, but also is easy to operate with few side effects.

INPLASY registration number: This protocol was registered with the International Platform of Registered Systematic Review and Meta-Analysis Protocols (INPLASY) on 21 October 2020 and was last updated on 21 October 2020 (registration number INPLASY2020100074).

\section{INTRODUCTION}

Review question / Objective: To evaluate the effect of acupuncture versus nonacupuncture treatment for rectal prolapse patients.

Condition being studied: Rectal prolapse is a kind of anorectal, rectal and partial sigmoid colon prolapse out of the anus disease, prone to children and the elderly, clinical incidence is not high, but because it is often accompanied by bleeding, edema, resulting in patients with fatigue, dizziness, and even incontinence, seriously affecting the quality of work and life.At present in view of the Rectal prolapse III degree and above, with the major surgery, and for 
Idegree, II degree mainly uses the conservative treatment in patients with Rectal prolapse. As western medicine has no specific treatment drugs, Treatment is symptomatic treatment, so the efficacy is poor, and the recurrence rate is high. Traditional Chinese medicine (TCM) has rich experience in treating Rectal prolapse, so many patients begin to seek TCM treatment. With the application of clinical practice, acupuncture, as a traditional green therapy, not only has remarkable curative effect, but also is easy to operate with few side effects.

\section{METHODS}

Participant or population: Patients with Rectal Prolapse.

Intervention: Acupuncture therapy, or acupunture combined with other treatments.

Comparator: Non-acupuncture leg: traditional Chinese medicine, Western medicine, placebo, sham acupuncture, no treatment, or any combination of these).

Study designs to be included: Randomized controlled trials (RCTs) which assessed the efficacy and safety of acupuncture for rectal prolapse will be included.

Eligibility criteria: Subjects: patients with rectal prolapse age, countries and The economic situation were not restricted. Intervention measures: acupuncture were used as the intervention measures in the treatment group, while non-acupunture therapy or traditional Chinese medicine was used as the intervention measures in the control group Outcome measures: cure rate, effective rate, inefficiency, recurrence rate, adverse reactions.

Information sources: Pubmed, Embase, Cochrane Library, Chinese Biomedical Literatures Database(CBM), China National Knowledge Infrastructure (CNKI), WangFang Database (WF), Chinese Scientifific Journal Database (VIP).
Main outcome(s): The cure rate, effective rate, inefficiency rate.

Additional outcome(s): Recurrence rate, adverse reactions.

Quality assessment / Risk of bias analysis: According to the improved Jadad scoring scale, the quality of the included literature was evaluated. 1-3 were classified as low quality and 4-7 as high quality. Risk of bias(quality) assessment Included randomised studies will be assessed for risk of bias by two independent raters(FWQ/YSB) using the Cochrane Collaboration's tool for assessing risk of bias in randomised trials. Any disagreements will be resolved through discussion or consultation with a third reviewer(YT).

Strategy of data synthesis: RevMan 5.4 software (Cochrane Collaboration) was used for the meta-analysis. Dichotomous data were reported as risk ratio (RR) with 95\% confidence intervals (CI), while continuous data were reported as standardized mean difference (SMD) with $95 \%$ Cls. The Higgins $I^{2}$ test was used to test heterogeneity with a significance level set at $50 \%$. If heterogeneity was not significant $\left(I^{2} \leq 50 \%\right)$, the fixed effects model was used for meta-analysis. Otherwise, the random effects model was used $\left(I^{2} \geq 50 \%\right)$. If possible, we investigated the potential explanations for heterogeneity and conducted subgroup analysis.

Subgroup analysis: If the necessary data are available, subgroup analysis will be carried out according to different factors as follows: 1.Control interventions (eg, traditional Chinese medicine, sham/ placebo acupuncture, no treatment, or non-TCM treatment). 2.Type of acupuncture and moxibustion(needle acupuncture, electronic-acupuncture, auricular acupuncture, heat-sensitive moxibustion, Thunder fire moxibustion, warm needling moxibustion, suspended moxibustion or mild moxibustion) 2.Outcome indicators (eg, recurrence rate, adverse reactions).

Sensibility analysis: Sensibility analysis: To assess the influence of each individual 
study, leave-one-out sensitivity analysis was performed iteratively by removing one study at a time to confirm that the findings were not influenced by any single study.

Country(ies) involved: China.

Keywords: Rectal Prolapse, Acupuncture.

Contributions of each author:

Author 1 - Wenqiang Fu - The author drafted the manuscript.

Author 2 - Yong Tang - The author provided statistical expertise.

Author 3 - Sibin Yi - The author contributed to the development of the selection criteria, and the risk of bias assessment strategy. 